Auffangnetze für ausgeschiedene Mandatsträger haben und dass das Übergangsgeld kaum seinem eigentlichen Zweck dient, könnten einen Impuls für die Diätendebatte geben, die unter dem Gesichtspunkt dieser Bedingungen der parlamentarischen Nachversorgung ganz anders geführt werden müsste. Zum Beispiel könnte darüber nachgedacht werden, ob Bundestagsabgeordnete in die sozialen Sicherungssysteme einzahlen, um nach dem Mandatsverlust einen Versorgungsschutz zu haben. Oder die Diäten könnten erhöht werden, um Mitgliedern des Bundestags den finanziellen Spielraum zu ermöglichen, sich für die nachparlamentarische Zeit privat zu versichern. Eine veränderte Versorgungsregelung könnte wiederum Auswirkungen auf die Rekrutierung des parlamentarischen Personals haben.

(4) Von enormer Bedeutung wäre zudem, den Verbleib ehemaliger Abgeordneter international vergleichend zu erforschen. Wie sind die rechtlichen Regelungen der Nachversorgung in den einzelnen Ländern gestaltet? Im Schweizer Milizsystem zum Beispiel ist ein reines Berufspolitikerdasein nicht vorgesehen. Wie vereinbaren Bundespolitiker in der Schweiz Beruf und Mandat? Wie schlägt sich das Milizprinzip in der rechtlichen Ausgestaltung politischer Ämter bis hin zur Nachversorgung nieder? In Großbritannien dürfen Angehörige des öffentlichen Dienstes nicht ins Parlament gewählt werden. Im Bundestag hingegen liegt der Anteil der Beamten und Angestellten des öffentlichen Dienstes bei knapp 45 Prozent der Abgeordneten. Diese Gruppe ist hinsichtlich der parlamentarischen Nachversorgung keinerlei Risiko ausgesetzt. Wie sieht die berufssoziologische Zusammensetzung des britischen Parlaments ohne Angehörige des öffentlichen Dienstes aus? Wie ist die Nachversorgung dort geregelt, und gibt es „Reaktionen“ der Abgeordneten oder der Parteien auf das Risiko einer gescheiterten Wiederwahl?

\title{
Der „Hammelsprung“ im Deutschen Bundestag
}

Kai Zähle

Kaum eine parlamentarische Übung erfreut sich so großer allgemeiner öffentlicher Aufmerksamkeit wie der so genannte Hammelsprung. Dies liegt nicht zuletzt an der außergewöhnlichen Bezeichnung dieses Abstimmungsverfahrens, welches in $₫ 51$ Abs. 2 der Geschäftsordnung des Bundestages (GO BT) geregelt ist: „Nachdem die Mitglieder des Bundestages auf Aufforderung des Präsidenten den Sitzungssaal verlassen haben, werden die Türen bis auf drei Abstimmungstüren geschlossen. An jeder dieser Türen stellen sich zwei Schriftführer auf. Auf ein Zeichen des Präsidenten betreten die Mitglieder des Bundestages durch die mit ,Ja', ,Nein’ oder ,Enthaltung' bezeichnete Tür wieder den Sitzungssaal und werden von den Schriftführern laut gezählt.“ Der Begriff „Hammelsprung“ bedeutet in der Parlamentspraxis der Bundesrepublik also die Auszählung der Stimmen

1 Peter Schindler, Datenhandbuch zur Geschichte des Deutschen Bundestages 1983-1991, BadenBaden 1994, S. 576, S. 581. 


\section{Herkunft des Begriffs}

Die Auszählung der Stimmen mittels Eintreten durch eine Tür hat eine jahrhundertealte parlamentarische Geschichte. Der Reichstag führte dieses Verfahren 1874 in seine Geschäftsordnung ein ${ }^{2}$. Im Preußischen Abgeordnetenhaus wurde der Begriff „Hammelsprung“ bereits 1885 verwandt ${ }^{3}$. Im 1894 fertig gestellten Reichstagsgebäude wurde über der „Ja“-Tür im Plenarsaal ein Intarsienbild aufgehängt, auf welchem ein Schafhirte seine Herde zählte - er ließ die Schafe zwischen seinen Beinen durchlaufen -, und über der „Nein“-Tür war der schlesische Berggeist Rübezahl in Holzschmuck beim Zählen seiner Rüben ausgearbeitet ${ }^{4}$. Der Architekt des Reichtagsgebäudes, Paul Wallot, verwandte bei der Inneneinrichtung des Hauses zahlreiche Kunstwerke, um die kulturelle Vielfalt Deutschlands zu dokumentieren und historische Ereignisse darzustellen ${ }^{5}$. Durch die Ausschmückung des Reichstages sollten auch Ideen des Parlamentarismus in Kunstwerken versinnbildlicht werden ${ }^{6}$. Also lag es nahe, über den Abstimmungstüren im Plenarsaal solche Kunstgegenstände zu verwenden, die einen Bezug zum Zählen aufwiesen. Deshalb kann die Aussage, das Abstimmungsverfahren werde wegen des Intarsienbildes „Hammelsprung“ genannt $^{7}$, keine Geltung beanspruchen. Auch wenn dieses Bild eng mit der Begriffsgeschichte verbunden ist, war es aufgrund des parlamentarischen Sprachgebrauches nicht Auslöser für die Bezeichnung des Abstimmungsverfahrens.

Die Szene des seine Schafe zählenden Hirten hat ihren Ursprung in der von Homer in der „Odyssee“ beschriebenen griechischen Mythologie ${ }^{8}$. In dieser überlistete Odysseus den geblendeten Polyphem beim Flüchten aus dessen Höhle, indem er sich an einen Schafsbauch klammerte, als Polyphem die Tiere durch seine Beine aus der Höhle ließ? 9

2 H. Jäckel, Stichwort Hammelsprung, in: Hans Röhring / Kurt Sontheimer (Hrsg.), Handbuch des deutschen Parlamentarismus, München 1970, S. 189.

3 Abg. Ernst von Eynern, Rede, Verhandlungen Preußisches Abgeordnetenhaus, 5. Legislaturperiode 3. Session, 52. Sitzung am 15. April 1885, S. 1349.

4 Abg. Paul Löbe zitiert bei Heinrich Georg Ritzel / Joseph Bücker / Hermann Josef Schreiner, Handbuch für die Parlamentarische Praxis, Frankfurt am Main, $\$ 51$ Anm. II a (September 2000); siehe auch Maximilian Rapsilber, Das Reichstagsgebäude, 4. Auflage, Leipzig 1913, S. 70 f. Die buntfarbige Holzintarsie (vgl. ders., Das Reichstagshaus in Berlin, Berlin 1894, S. 40) von Ernst Nast ist abgebildet bei Michael S. Cullen, Der Reichstag, 2. Auflage, Berlin 1999, S. 154. Rübezahl ist eine Sagengestalt, um die sich mehrere Volkserzählungen ranken. Nach einer Sage soll der schlesische Berggeist des Riesengebirges dadurch zu seinem Namen gekommen sein, indem er für eine von ihm gefangen gehaltene Prinzessin Rüben züchten und zählen musste.

5 Siehe zur Formensprache von Wallot: Tillmann Buddensieg, in: Heinrich Wefing (Hrsg.), „Dem Deutschen Volke“, Bonn 1999, S. 30 (S. 36); Uwe Kieling, Der Deutsche Reichstag, Berlin 1995, S. 63.

6 Michael S. Cullen, Der Reichstag, Berlin 1983, S. 275.

7 Martin Lohmeier, Bundestag A bis Z, Bonn 1983, S. 7; R. Peter Dach, in: Bonner Kommentar zum Grundgesetz, Heidelberg, Art. 40 Rn. 99 (Oktober 1996).

8 Michael F. Feldkamp, Datenhandbuch zur Geschichte des Deutschen Bundestages 1994-2003, Baden-Baden 2005, S. 420; Creifelds, Rechtswörterbuch, 18. Auflage, München 2004, Stichwort Hammelsprung, S. 629; Tobias Abeßer, Eine parlamentarische Odyssee, in: Jura 1994, S. 559.

9 Homer, Odyssee, 9. Gesang, Zeilen 345 - 397. Der Text wurde um etwa 750/730 v. Chr. verfasst, Marion Giebel (Hrsg.), Homer Odyssee Auswahl, Stuttgart 2004, S. 6. 


\section{Entstehungsgeschichte des $\$ 51$ Abs. 2 GO BT und die historische Entwicklung}

Die Entstehungsgeschichte der Abstimmung durch Auszählen der Stimmen nach $\$ 51$ Abs. 2 GO BT reicht bis in die Anfangszeit des deutschen Parlamentarismus zurück ${ }^{10}$. Ursprünglich wurde dieses Abstimmungsverfahren aus der Geschäftsordnung des britischen Unterhauses entnommen ${ }^{11}$ und bereits in der Frankfurter Nationalversammlung durchgeführt.

\subsection{Von der Frankfurter Nationalversammlung bis zum Reichstag}

In der Frankfurter Nationalversammlung 1848/1849 hatten die Abgeordneten Namenszettel, auf denen sie „Ja“ oder „Nein“ schrieben; die Zettel wurden anschließend durch Sekretäre eingesammelt und gezählt. Damals war aber oft zweifelhaft, ob nur Anwesende gestimmt hatten oder ob für Abwesende mitgestimmt worden war ${ }^{12}$. In den Geschäftsordnungen des Preußischen Landtags vom 28. März 1849 und 6. Juni 1862 erfolgte die Zählung der Stehenden und der Sitzenden. Während der Zeit des Norddeutschen Bundes von 1867 bis 1870 sah dessen Geschäftsordnung vom 12. Juni 1868 vor, dass der Präsident mit der Glocke das Zeichen zum Verlassen des Saals gab und die Abgeordneten diesen durch zwei Türen wieder betreten sollten. Die zwei Türen waren zwar nicht mit „Ja“ und „Nein“ überschrieben, deren Position wurde gleichwohl genau beschrieben: Die „Ja“-Tür befand sich an der Ost-Seite und die „Nein“-Tür an der West-Seite des Sitzungssaales. Dieses Verfahren ähnelte zum ersten Mal dem heute praktizierten Verfahren ${ }^{13}$.

Nach der Reichsgründung wurde diese Regelung übernommen. Am 9. April 1874 brachte der Abgeordnete Hans Victor von Unruh den Antrag ein, die Auszählung des Hauses dadurch zu bewirken, dass die Abgeordneten den Sitzungssaal verlassen und beim jeweiligen Eintreten durch die „Ja“- beziehungsweise „Nein“-Tür bei den Schriftführern einen Namenszettel abgeben ${ }^{14}$. Diese Abstimmungsart sollte auch die namentliche Abstimmung ersetzen; ein Namensaufruf zum Zwecke der Abstimmung sollte nicht mehr stattfinden ${ }^{15}$. In der Debatte stellte der Abgeordnete Franz August Freiherr Schenk von Stauffenberg den Änderungsantrag, dass die Abgeordneten lediglich durch die entsprechenden Türen gingen; auf die

10 Siehe hierzu die synoptische Darstellung zu $\$ 51$ GO BT in: Deutscher Bundestag (Hrsg.), Die Geschäftsordnungen deutscher Parlamente seit 1848, Bonn 1986.

11 Abg. Hans Victor von Unruh, Rede, Verhandlungen des Deutschen Reichstages, 2. Legislaturperiode 1. Session, 27. Sitzung am 9. April 1874, S. 681; R. Schneider, in: Bonner Kommentar, a.a.O., Art. 42 Anm. 4 b.

12 Abg. Hans Victor von Unruh, Rede, Verhandlungen des Deutschen Reichstages, 2. Legislaturperiode 1. Session 1874, 27. Sitzung am 9. April 1874, S. 680.

13 Tobias Abeßer, a.a.O., S. 559.

14 Antrag der Abg. Hans Victor von Unruh und Genossen auf Aenderung der $\$ \$ 52$ und 54 der Geschäftsordnung bezüglich der namentlichen Abstimmung, Antrag vom 24. März 1874, Nr. 110 der Drucksachen, Aktenstücke zu den Verhandlungen des Deutschen Reichstages 1874; Debatte: Verhandlungen Deutscher Reichstag, 2. Legislaturperiode 1. Session, Sitzung am 9. April 1874, S. 680 - 687. Siehe hierzu auch Abg. Peter Spahn, Verhandlungen Deutscher Reichstag, 10. Legislaturperiode 2. Session, 215. Sitzung am 13. November 1902, S. 6340.

15 Vgl. August Plate, Geschäftsordnung des Preußischen Abgeordnetenhauses, 2. Auflage, Berlin 1904, S. 179. 
Abgabe von Namenszetteln wurde verzichtet ${ }^{16}$. Daraufhin beschloss der Reichstag, die Stimmen lediglich beim Betreten des Plenarsaals durch eine von zwei Türen zu zählen ${ }^{17}$.

Die in der Geschäftsordnung des Reichstages nicht vorgesehene Tür für Enthaltungen führte zu dem Problem, dass die Abgeordneten eigentlich keine Gelegenheit hatten, ihre Anwesenheit bei neutralem Verhalten zum Beschlussgegenstand zu dokumentieren. In der Parlamentspraxis bildete sich die Übung, dass sich diese Abgeordneten gesondert beim Präsidenten meldeten, etwa indem sie beim Heraustreten der abstimmungsbereiten Mitglieder im Sitzungssaal verblieben und dann den Präsidenten auf sich aufmerksam machten; dieser vermerkte dann im Stenographischen Bericht deren Enthaltung ${ }^{18}$.

Auch der Reichstag der Weimarer Republik übernahm diese Abstimmungsform. $\$ 104$ der Geschäftsordnung vom 12. Dezember 1922 erwähnt erstmals eine Tür für Enthaltungen ${ }^{19}$. Dadurch erhielten die Abgeordneten eine auch in der Geschäftsordnung normierte Möglichkeit, ihre Anwesenheit ohne Stimmabgabe zu bekunden ${ }^{20}$.

\subsection{Deutscher Bundestag}

Auf Empfehlung des Ausschusses für Geschäftsordnung und Immunität ${ }^{21}$ beschloss der Bundestag am 1. März 1950 die satzungsgemäße Einführung des „Hammelsprungs“ in $\$ 104$ seiner vorläufigen Geschäftsordnung ${ }^{22}$. Somit fand der „Hammelsprung“ Eingang in den Bundestag. Aus der Geschäftsordnungsentwicklung ist vor allem das Verhalten der Berliner Abgeordneten während der Auszählung der Stimmen bemerkenswert. Deren eingeschränktes Stimmrecht bis zur Wiedervereinigung 1990 führte beim „Hammelsprung“ dazu, dass sie während des Abstimmungsverfahrens regelmäßig im Sitzungssaal sitzen blieben ${ }^{23}$. Bis zum Ende der 15. Wahlperiode wurde der „Hammelsprung“ insgesamt 513-mal durchgeführt ${ }^{24}$.

16 Antrag vom 8. April 1874, Aktenstück 138 zu den Verhandlungen des Deutschen Reichstages 1874.

17 Verhandlungen des Deutschen Reichstages, 2. Legislaturperiode 1. Session, 27. Sitzung am 9. April 1874, S. 687.

18 Vgl. Bernhard Jungheim, Geschäftsordnung für den Reichstag, Berlin 1916, S. 205 ff.; Kurt Perels, Das autonome Reichstagsrecht, Berlin 1903, S. 83; Heinrich Georg Ritzel / Joseph Bücker / Hermann Josef Schreiner, a.a.O., $\$ 51$ Anm. II c (September 2000).

19 Tobias Abeßer, a.a.O., S. 559.

20 Abg. Paul Löbe zitiert bei Heinrich Georg Ritzel / Joseph Bücker / Hermann Josef Schreiner, a.a.O., $\$ 51$ Anm. II a (September 2000).

21 Mündlicher Bericht des Ausschusses für Geschäftsordnung und Immunität auf Änderung des $\$ 104$ der vorläufigen Geschäftsordnung des Deutschen Bundestages, BT-Drs. 1/528.

22 Siehe hierzu die Rede des Abg. Heinrich Georg Ritzel vom 1. März 1950, Stenographische Protokolle Deutscher Bundestag, 1/43, S. $1459 \mathrm{f}$.

23 Peter Schindler, Datenhandbuch zur Geschichte des Deutschen Bundestages 1949-1982, 3. Auflage, Baden-Baden 1984, S. 798 f. Auslöser dieser Praxis war offenbar ein Vorstoß des Abg. Carlo Schmid (Frankfurt) auf einen Vorschlag des Vizepräsidenten Karl Mommer innerhalb einer kontroversen Abstimmung 1969, Stenographische Protokolle Deutscher Bundestag, 5/222, S. 12066. Zur Problematik des Verlassens des Sitzungssaales während eines laufenden „Hammelsprungverfahrens" siehe Abg. Herwart Miessner und Abg. Paul Löbe, Stenographische Protokolle Deutscher Bundestag, 1/43, S. 1460.

24 Peter Schindler, Datenhandbuch zur Geschichte des Deutschen Bundestages 1949-1999, BadenBaden 1999, S. 3690; Michael F. Feldkamp, a.a.O., S. 420; ders., Deutscher Bundestag 1987 bis 2005: Parlaments- und Wahlstatistik, in: ZParl, 37. Jg. (2006), H. 1, S. 17. 


\section{Verfahren des „Hammelsprungs“}

\subsection{Einordnung innerhalb der Abstimmungsarten}

Das Parlamentsrecht kennt unterschiedliche Abstimmungsarten ${ }^{25}$. Diese lassen sich der Form und der Offenkundigkeit nach unterscheiden. Der Form nach gibt es Abstimmungen durch Zeichen - etwa durch Handzeichen oder durch Aufstehen oder Sitzenbleiben $(\$ 48$ GO BT) - und die schriftliche Abstimmung ( $\$ \$ 49$, 52 GO BT mit Stimmzettel beziehungsweise mit Abstimmungskarten). Nach der Offenkundigkeit lassen sich geheime - wie die Wahl mit verdeckten Stimmzetteln ( $\$ 49$ GO BT) - und offene Abstimmungen - wie Handzeichen, Aufstehen oder Sitzenbleiben - unterscheiden ${ }^{26}$. Die meisten Abstimmungen finden durch Handzeichen statt. Bei der Schlussabstimmung über Gesetzentwürfe wird jedoch nach $\$ 48$ Abs. 1 Satz 2 GO BT durch Aufstehen und Sitzenbleiben abgestimmt. Diesen Abstimmungsarten ist gemeinsam, dass die Abgeordneten während des gesamten Verfahrens im Plenarsaal verbleiben und das Abstimmungsergebnis nach der Zusammensetzung von Mehrheit und Minderheit geschätzt wird. Einen Sonderfall stellt der „Hammelsprung" dar, weil die Abgeordneten den Saal zunächst verlassen und beim Betreten des Sitzungssaales einzeln gezählt werden. Der „Hammelsprung“ ist eine offene Abstimmung durch Zeichen, weil beim Hereintreten in den Plenarsaal erkennbar ist, wie die Abgeordneten abstimmen.

Korrelat zum „Hammelsprung“ ist die namentliche Abstimmung ( $\$ 52$ GO BT), welche die Auszählung der Stimmen ersetzt ${ }^{27}$. Dies ergibt sich aus den $\$ \$ 45$ Abs. 2 Satz 1, 91 Satz 3 GO BT. Insbesondere $\$ 91$ Satz 3 GO BT verdeutlicht den Vorrang der namentlichen Abstimmung vor der Abstimmung durch Zählung (,Zählung der Stimmen, wenn nicht namentliche Abstimmung verlangt wird“). Auch bei der namentlichen Abstimmung werden die Stimmen gezählt. Im Unterschied zum „Hammelsprung“ kann jedoch die Stimmabgabe den jeweiligen Abgeordneten zugeordnet werden, weil sie in den Plenarprotokollen festgehalten wird ${ }^{28}$. Das Stimmverhalten verbleibt also nicht anonym. In der Praxis hat die namentliche Abstimmung den „Hammelsprung“ faktisch abgelöst. Nachdem der „Hammelsprung“ in den ersten beiden Wahlperioden 181-mal (1949-53) und 121-mal (1953-57) erfolgte, betrug die Anzahl von der 10. bis zur 15. Wahlperiode 34 (10. WP (1983-87) 10, 11. WP (1987-90) 4, 12. WP (1990-94) 4, 13. WP (1994-98) 6, 14. WP (1998-2002) 6, 15. WP (2002-2005) 4) ${ }^{29}$. Demgegenüber erfolgte allein in diesem Zeitraum die namentliche Abstimmung 868-mal (10. WP: 128, 11. WP: 162, 12. WP: 129, 13. WP: 177, 14. WP: 170, 15. WP: 102) ${ }^{30}$. Es ist zu vermuten, dass diese Entwicklung letztlich auf dem Vorteil der namentlichen Abstimmung - Protokollierung des Stimmverhaltens - basiert.

25 Vgl. dazu auch die von Michael F. Feldkamp editierte Rede von Paul Löbe in diesem Heft der ZParl.

26 Vgl. hierzu Norbert Achterberg, Parlamentsrecht, Tübingen 1984, S. 641.

27 Ebenda, S. 646; Hans Trossmann, Parlamentsrecht und Praxis des Deutschen Bundestages, Bonn 1967, S. 46.

28 Vgl. Peter Schindler, 1999, a.a.O., S. 1726.

29 Ebenda, S. 3690; Michael F. Feldkamp, 2005, a.a.O, S. 420; ders., 2006, a.a.O., S. 17.

30 Siehe Peter Schindler, 1999, a.a.O., S. 1728; Michael F. Feldkamp, 2005, a.a.O., S. 421; ders., 2006, a.a.O., S. 17. 


\subsection{Anwendungsbereich}

Die GO BT kennt drei normierte Anwendungsfälle des „Hammelsprungs“: die Bezweifelung der Beschlussfähigkeit des Bundestages nach $\$ 45$ Abs. 2 GO BT, die Uneinigkeit des Sitzungsvorstandes über das Abstimmungsergebnis nach $\$ 51$ Abs. 1 GO BT sowie die Zurückweisung eines Einspruches des Bundesrates gegen Gesetzesbeschlüsse des Bundestages nach $\$ 91$ GO BT. Hinzu kommt noch die parlamentarische Übung bei ungeregelten Fällen.

(1) $\$ 45$ Abs. 2 Satz 1 GO BT: Grundsätzlich wird die Beschlussfähigkeit des Bundestages fingiert, indem unterstellt wird, dass stets mehr als die Hälfte seiner Mitglieder im Sitzungssaal anwesend ist. Nach $\$ 45$ Abs. 2 Satz 1 GO BT ist ein „Hammelsprung“ allerdings dann durchzuführen, wenn vor Beginn einer Abstimmung die Beschlussfähigkeit von einer Fraktion oder von anwesenden fünf von Hundert der Abgeordneten bezweifelt und auch vom Sitzungsvorstand nicht einmütig bejaht wird oder die Beschlussfähigkeit vom Sitzungsvorstand im Einvernehmen mit den Fraktionen bezweifelt wird. Die Feststellung der Beschlussfähigkeit erfolgt hierbei gleichzeitig mit der Abstimmung. Im Laufe einer Kernzeitdebatte (Donnerstagsdebatte) ist die Beschlussfähigkeit indes durch eine namentliche Abstimmung nach $₫ 52$ GO BT festzustellen. Jedoch kann eine namentliche Abstimmung nicht mehr angeordnet werden, wenn die Gegenprobe nach $₫ 51$ Abs. 1 Satz 1 GO BT durchgeführt wird, weil die Gegenprobe ein Teil der Abstimmungshandlung ist ${ }^{31}$.

(2) $\$ 51$ Abs. 1 GO BT: Nach dieser Vorschrift werden die Stimmen gezählt, wenn der Sitzungsvorstand über das Ergebnis einer Abstimmung auch nach Durchführung der Gegenprobe nicht einig ist. Dies kann sich beispielsweise dann ergeben, wenn die Abgeordneten durch Handzeichen oder durch Aufstehen oder Sitzenbleiben ihre Stimme abgegeben haben und sich kein eindeutiges Meinungsbild ergeben hat.

(3) $\$ 91$ GO BT: Nach dieser Vorschrift wird über den Antrag auf Zurückweisung eines Einspruches des Bundesrates gegen ein vom Bundestag beschlossenes Gesetz durch Zählung der Stimmen abgestimmt, wenn nicht die namentliche Abstimmung verlangt wird. Nach $\$ 52$ Satz 1 GO BT kann eine namentliche Abstimmung bis zur Eröffnung der Abstimmung von einer Fraktion oder von anwesenden fünf von Hundert der Mitglieder des Bundestages verlangt werden.

(4) Ständige Übung: Im Bundestag ist es auch ständige Übung, einen „Hammelsprung“ durchzuführen, wenn eine durch Grundgesetz, Bundesgesetz oder GO BT erforderliche qualifizierte Mehrheit für einen Beschluss oder eine Wahl zu erreichen ist und dies nicht anderweitig festgestellt werden $\mathrm{kann}^{32}$. Letzteres ist etwa bei einer namentlichen Abstimmung der Fall.

31 Heinrich Georg Ritzel / Joseph Bücker / Hermann Josef Schreiner, a.a.O., $\$ 51$ Anm. I 1 b, 2 a (September 2000).

32 Hans Trossmann, Parlamentsrecht des Deutschen Bundestages, München 1977, $₫ 56$ Rn. 1; Peter Schindler, 1994, a.a.O., S. 581; Hans-Achim Roll, Geschäftsordnung des Deutschen Bundestages, Baden-Baden 2001, $\$ 51$ Rn. 1. 


\subsection{Durchführung}

Der „Hammelsprung“ wird in fünf Schritten durchgeführt.

(1) Anordnung des Sitzungsvorstandes ( $\$ 51$ Abs. 1 Satz 3 GO BT): Die Auszählung der Stimmen kann vom Sitzungsvorstand - amtierender Bundestagspräsident, das heißt auch der Vizepräsident, und zwei Schriftführer ( $\$ 8$ Abs. 1 GO BT) - angeordnet werden, wenn der Anwendungsbereich des „Hammelsprungs“ eröffnet ist. Er kann nicht von einzelnen Abgeordneten oder einer Fraktion beantragt werden ${ }^{33}$. Das Fehlen dieser Möglichkeit entspricht auch dem Willen des historischen Satzungsgebers. In der Debatte über die Einführung des „Hammelsprungs“ in die vorläufige Geschäftsordnung beschloss der Bundestag auf Antrag der Fraktion der FDP, entgegen der Empfehlung des Geschäftsordnungsausschusses eine Beantragung des „Hammelsprungs“ durch mindestens 30 Abgeordnete nicht vorzusehen ${ }^{34}$.

Die Feststellung des Präsidenten, dass der Sitzungsvorstand zu keiner übereinstimmenden Meinung gekommen ist, ist nicht anfechtbar ${ }^{35}$. Die Uneinigkeit des Sitzungsvorstandes liegt auch dann vor, wenn ein Schriftführer ein Abstimmungsergebnis entgegen der Ansicht des Präsidenten beurteilt ${ }^{36}$. Dies ergibt sich aus $\$ 45$ Abs. 2 Satz 1 GO BT, wonach der Sitzungsvorstand die Beschlussfähigkeit einmütig beurteilen muss. Der Präsident kann demzufolge die Schriftführer nicht überstimmen.

(2) Aufforderung des Präsidenten zum Verlassen des Sitzungssaales und Schließen der Sitzungstüren ( $\$ 51$ Abs. 2 Sätze 1, 2 GO BT): Anschließend bittet der Präsident die Abgeordneten, den Sitzungssaal zu verlassen. Danach werden die Türen bis auf die drei Abstimmungstüren geschlossen. An jeder dieser Türen stellen sich zwei Schriftführer auf, je einer aus der Regierungskoalition und der Opposition.

(3) Zeichen des Präsidenten zum Betreten des Sitzungssaales und lautes Zählen durch die Schriftführer ( $\$ 51$ Abs. 1 Satz 3 GO BT): Auf ein Zeichen des Präsidenten - etwa mit der Glocke - betreten die Abgeordneten jeweils durch eine der mit „Ja“, „Nein“ oder „Enthaltung" bezeichneten Türen wieder den Sitzungssaal und werden dabei von den Schriftführern laut gezählt. Sollte ein Abgeordneter beim „Hammelsprung“ versehentlich die unrichtige Abstimmungstür benutzt haben, ist dies unbeachtlich ${ }^{37}$, weil nach $₫ 46$ GO BT das Thema einer Abstimmung grundsätzlich eindeutig ist. Der Präsident stellt die Fragen so, dass sie sich mit „Ja“ oder „Nein“ beantworten lassen. Die Stimmabgabe kann mithin nicht angefochten werden.

In der Parlamentspraxis ist ein anschaulicher Fall zur Benutzung der unrichtigen Abstimmungstür dokumentiert. In der 4. Wahlperiode stimmte der Abgeordnete Hans Richarts (CDU/CSU) in Zusammenhang mit der Spiegel-Affäre irrtümlicherweise für einen Antrag

33 Hans Trossmann, 1977, a.a.O., $\$ 56$ Rn. 4 mit Verweis auf: Stenographische Protokolle Deutscher Bundestag, 1/43, S. 1460 vom 1. März 1950.

34 Stenographische Protokolle Deutscher Bundestag, 1/43, S. 1459 f.

35 Hans Trossmann, 1977, a.a.O., $\$ 56$ Rn 3 mit Verweis auf: Stenographische Protokolle Deutscher Bundestag, 1/109, S. 4150 vom 10. Januar 1951.

36 Heinrich Georg Ritzel / Joseph Bücker / Hermann Josef Schreiner, a.a.O., \$51 Anm. I 1 c (September 2000).

37 Hans Trossmann, 1977, a.a.O., $\$ 56$ Rn 6; siehe bereits Reichstagspräsident Maximilian Franz August von Forckenbeck, Verhandlungen des Deutschen Reichstages, 2. Legislaturperiode 2. Session, 55. Sitzung am 28. Januar 1875, S. 1388. 
der oppositionellen SPD. Noch während der Abstimmung machte Richarts die beiden Schriftführer am Mitteleingang darauf aufmerksam und bat den Vizepräsidenten Richard Jaeger, diesen Irrtum zu korrigieren. Dies wurde von Jaeger abgelehnt ${ }^{38}$. Damit solche Fälle vermieden werden, stellen sich die Parlamentarischen Geschäftsführer der Fraktionen in der Praxis zum Teil an derjenigen Tür auf, die bei der Abstimmung benutzt werden sollte ${ }^{39}$.

Fraglich ist, ob ein Abgeordneter oder eine Gruppierung beziehungsweise eine Fraktion von Abgeordneten dem „Hammelsprung“ fernbleiben kann, um die Beschlussunfähigkeit des Bundestags absichtlich zu erreichen und eine wirksame Abstimmung zu verhindern ${ }^{40}$. Nach $\$ 13$ Abs. 2 Satz 1 GO BT sind die Mitglieder des Bundestages verpflichtet, an den Arbeiten des Bundestages teilzunehmen, wobei allerdings $₫ 13$ Abs. 2 Satz 3 GO BT nur vorsieht, bei der Nichtbeteiligung an einer namentlichen Abstimmung indirekten Zwang auszuüben. $\$ 14$ Abs. 2 AbgG schreibt in solchen Fällen die Kürzung der monatlichen Kostenpauschale um 50 Euro vor. $\$ 13$ Abs. 2 GO BT schafft indes keine durchsetzbare Verpflichtung, an den Arbeiten des Bundestages teilzunehmen ${ }^{41}$. Die Abgeordneten üben nach Art. 38 Abs. 1 Satz 2 GG ein freies Mandat aus. Hierbei wird davon ausgegangen, dass sie ihren Rechtspflichten ordnungsgemäß nachkommen. Es kann ihnen nicht vorgeschrieben werden, wie sie ihre Amtspflichten zu erfüllen haben. Insofern trifft sie lediglich die moralische, nicht durchsetzbare Pflicht, auch tatsächlich an der Auszählung der Stimmen teilzunehmen. Aus $\$ 31$ Abs. 2 GO BT ergibt sich überdies, dass die Abgeordneten frei sind, an Abstimmungen teilzunehmen, denn jeder kann vor einer Abstimmung die Erklärung abgeben, dass er nicht an der Abstimmung teilnimmt. Die Abgeordneten haben also auch das Recht, einer Abstimmung absichtlich fernzubleiben. Die Verpflichtung zur Teilnahme am „Hammelsprung“ wäre daher verfassungswidrig ${ }^{42}$.

(4) Zeichen des Präsidenten zur Beendigung der Zählung (\$51 Abs. 2 Satz 4 GO BT): Zur Beendigung der Auszählung gibt der Präsident ein Zeichen, und er selbst sowie die Schriftführer stimmen öffentlich ab - etwa durch lautes Sagen. Abgeordnete, die später eintreten, etwa weil sie nicht binnen angemessener Zeit von ihren Büros in den Plenarsaal gegangen sind, werden nicht mitgezählt ( $\$ 51$ Abs. 2 Sätze 4 bis 6 GO BT). In der parlamentarischen Praxis vergewissern sich die Schriftführer, ob sich noch weitere Abgeordnete vor den Abstimmungstüren befinden, die den Sitzungssaal zur Zählung ihrer Stimme betreten wollen. Des Weiteren hat das Präsidium mit Zustimmung des Ältestenrats in seinen im Jahr 2000 beschlossenen Leitlinien zum Abstimmungsverfahren nach $\$ 51$ GO BT 43 festgelegt, dass die Auszählung nach 20 Minuten beendet werden kann. Der Präsident kann auch mit den Fraktionen Kontakt aufnehmen, ob die Abstimmungszeit auf Grund außergewöhnlicher Umstände zu verlängern ist; dies ist vorstellbar, wenn parallel eine Ausschusssitzung stattfindet.

38 Abg. Hans Richarts (CDU/CSU), Erklärung vom 28. Juni 1962, Stenographische Protokolle Deutscher Bundestag, 4/37, S. 1584.

39 Ergebnis einer Anfrage an die Büros der Parlamentarischen Geschäftsführer der im Bundestag vertretenen Fraktionen vom Juli 2006.

40 Siehe bereits Abg. Adolf Gröber, Rede, Verhandlungen des Deutschen Reichstages, 9. Legislaturperiode 4. Session, 230. Sitzung am 24. Mai 1897, S. 6053.

41 Heinrich Georg Ritzel / Joseph Bücker / Hermann Josef Schreiner, a.a.O., $\$ 13$ Anm. II 1 a (Juli 1993); Hans-Achim Roll, a.a.O., $\$ 13$ Rn. 2.

42 Vgl. Hans Trossmann, 1977, a.a.O., $\$ 16$ Rn. 2 zur namentlichen Abstimmung.

43 Leitlinien vom 5. April 2000, abgedruckt bei Heinrich Georg Ritzel / Joseph Bücker / Hermann Josef Schreiner, a.a.O., \$51 Anm. II d (September 2000). 
(5) Verkündung des Abstimmungsergebnisses durch den Präsidenten ( $\$ 51$ Abs. 2 Satz 7 GO BT): Schließlich verkündet der Präsident nach $\$ 51$ Abs. 2 Satz 7 GO BT das Ergebnis der Abstimmung. Dieser Beschluss ist nach dem gewohnheitsrechtlichen Grundsatz der Unverrückbarkeit der Beschlüsse weder aufhebbar noch veränderbar ${ }^{44}$.

\subsection{Exkurs: Geschäftsordnungen der Landesparlamente und des Bundesrates}

Auch zahlreiche Parlamente in den Bundesländern sehen in ihren Geschäftsordnungen den „Hammelsprung “ vor. In sieben Landtagen ist dieses Verfahren bei der Uneinigkeit über das Abstimmungsergebnis normiert, wobei der Begriff zweimal wörtlich verwandt wird ${ }^{45}$. In sechs Geschäftsordnungen wird bei diesem Anwendungsfall lediglich die Zählung der anwesenden Abgeordneten ohne eine nähere Beschreibung des Verfahrens angeordnet ${ }^{46}$. Als Sonderfall sieht die baden-württembergische Geschäftsordnung den Namensaufruf vor (\$97 Abs. 1 Satz 3).

Bei der Bezweifelung der Beschlussfähigkeit wird in elf Landtagen die Zählung der anwesenden Abgeordneten vorgeschrieben, wobei zweimal eine Wahl zwischen dem Namensaufruf und der Auszählung - auch hier wird das Verfahren nicht näher beschrieben eingeräumt wird ${ }^{47}$. In vier Geschäftsordnungen ist in diesen Fällen jedoch der Namensaufruf obligatorisch vorgesehen ${ }^{48}$.

Die Geschäftsordnung des Bundesrates kennt den Hammelsprung nicht ${ }^{49}$.

\section{Ersatz durch ein elektronisches Abstimmungsverfahren?}

Seitdem der „Hammelsprung“ in die parlamentarischen Geschäftsordnungen aufgenommen wurde, wird darüber diskutiert, ihn durch ein elektrisches beziehungsweise elektronisches Abstimmungsverfahren zu ersetzen oder zu ergänzen.

\subsection{Entwürfe von Werner Siemens für das Preußische Abgeordnetenhaus beziehungsweise den Reichstag}

Für das Preußische Abgeordnetenhaus ersann das Unternehmen Telegraphen-Bauanstalt von Siemens \& Halske um 1859 einen Abstimmungstelegraphen, mit dem eine namentliche Abstimmung und die Auszählung der Stimmen binnen einer halben Minute ausge-

44 Siehe Hans-Achim Roll, a.a.O., $\$ 48$ Rn. 7.

45 Berlin $₫ 70$ Abs. 2, Hamburg $\$ 34$ Abs. 4, Niedersachsen $₫ 83$ Abs. 3, Sachsen $₫ 103$ Abs. 3, Sachsen-Anhalt $\$ 74$ Abs. 3, sowie wörtliche Verwendung in Bayern $\$ 129$ und Nordrhein-Westfalen $\$ 42$ Abs. 5.

46 Brandenburg $\$ 67$ Abs. 2, Bremen $\$ 57$ Abs. 2 Satz 1, Hessen $\$ 83$ Abs. 4, Mecklenburg-Vorpommern $\$ 90$ Abs. 5, Rheinland-Pfalz $\$ 43$ Abs. 5, Thüringen $\$ 41$ Abs. 5.

47 Berlin $\$ 73$ Abs. 1 Satz 2, Brandenburg $\$ 63$ Abs. 2, Hamburg $\$ 32$ Abs. 3 Satz 3, Hessen $\$ 61$ Abs. 1, Mecklenburg-Vorpommern $₫ 77$ Abs. 2, $\$ 18$ Abs. 1 Satz 2, Rheinland-Pfalz $₫ 42$ Abs. 2, Saarland $₫ 47$, Sachsen $₫ 83$ Abs. 2, $\$ 35$ Abs. 1, Thüringen $₫ 40$ Abs. 2; Wahlmöglichkeit in Nordrhein-Westfalen $\$ 39$ Abs. 3 und Schleswig-Holstein $₫ 59$ Abs. 2.

48 Baden-Württemberg $₫ 80$ Abs. 1, Bayern $₫ 123$ Abs. 2, Niedersachsen $₫ 79$ Abs. 3 Satz 1, Sachsen-Anhalt $\$ 70$ Abs. 3.

49 Vgl. Konrad Reuter, Praxishandbuch Bundesrat, 2. Auflage, Heidelberg 2007, \$29 GO Rn. 3. 
führt werden sollte ${ }^{50}$. Hierzu entwickelte Werner Siemens eine Maschine, bei der auf jedem Abgeordnetenplatz eine Kurbel mit einem Schloss installiert wurde (rechte Stellung für „Ja“, linke Stellung für „Nein“; die mittige Stellung hatte keine Auswirkungen); mit einem individualisierten Schlüssel für das Schloss hatten sich die Abgeordneten zu identifizieren. Nach der Aufforderung des Präsidenten an die Abgeordneten zur Stimmabgabe sollte ein Saaldiener die Kurbel eines Stromgebers drehen. Alle Ströme bewegten drei Zeiger: je einen für „Ja“ und „Nein“; ein gemeinschaftlicher Zeiger diente zur Kontrolle der Summe der abstimmenden Abgeordneten ${ }^{51}$.

Letztlich nahmen die Abgeordneten hiervon nicht aus praktischen, sondern aus parlamentarisch-politischen Gründen Abstand ${ }^{52}$. Damals wurde die Auffassung vertreten, „die persönliche Abstimmung sei eine wichtige Bedingung für die Frische und Gesundheit des parlamentarischen Lebens und könne im Interesse der Parteidisziplin und der wirksamen Führung der Geschäfte einer großen, mit bedeutenden Aufgaben betrauten Körperschaft nicht entbehrt werden" 53 . In der Sitzung des Reichstages am 9. April 1874 stellte der Abgeordnete Leopold Graf von Frankenberg den Antrag, ebenfalls die Firma Siemens \& Halske mit der Entwicklung und der probeweisen Installation eines Abstimmungstelegraphen zu beauftragen ${ }^{54}$. Aus dem Brief von Werner Siemens an F. Lages ergibt sich, dass dieser Plan aus denselben Gründen wie 1859 nicht verwirklicht wurde.

\subsection{Erfahrungen im Bundestag}

In der Geschäftsordnungsdebatte des Bundestages über die Einführung des „Hammelsprungs“ am 1. März 1950 berichtete der Abgeordnete Heinrich Georg Ritzel von Überlegungen des Präsidenten Erich Köhler, eine technische Abstimmungsanlage in den Sitzungssaal einbauen zu lassen, wodurch sich der „Hammelsprung“ erübrigen würde ${ }^{55}$. Der Einbau fand jedoch keine Zustimmung ${ }^{56}$.

Zu Ostern 1970 wurde im Plenarsaal dann der Abstimmungscomputer „AEG 60-10Digitalrechner" eingebaut ${ }^{57}$. Hierzu wurden am Pult des Präsidenten der Leitstand des Computers errichtet, die Sitzplätze der Abgeordneten mit einer Knopfdruckanlage ausgestattet und an ihren Stühlen Kontakte installiert, die sich ab einer Belastung von 40 Kilo-

50 August Plate, a.a.O., S. 184.

51 Siehe hierzu Brief von Werner Siemens an seinen Bruder Wilhelm vom 18. Januar 1859, der von Siemens Corporate Archives freundlicherweise dem Verfasser zur Verfügung gestellt wurde; Werner Siemens, Wissenschaftliche und technische Arbeiten, Band 2, 2. Auflage, Berlin 1891, S. $307 \mathrm{ff}$.

52 Brief von Werner Siemens an Herrn F. Lages vom 27. Oktober 1882, der von Siemens Corporate Archives freundlicherweise dem Verfasser zur Verfügung gestellt wurde.

53 August Plate, a.a.O., S. $184 \mathrm{f}$.

54 Abg. Leopold Grafvon Frankenberg, Verhandlungen des Deutschen Reichstages, 2. Legislaturperiode 1. Session, 27. Sitzung am 9. April 1874, S. 683 f.; Antrag Verhandlungen des Deutschen Reichstages, 2. Legislaturperiode 1. Session 1874, Anlagen Band 37 Aktenstück Nr. 133.

55 Vgl. Abg. Heinrich Georg Ritzel, Rede am 1. März 1950, Stenographische Protokolle Deutscher Bundestag, 1/43, S. 1459.

56 Peter Schindler, 1999, a.a.O., S. 1727.

57 H. Jäckel, Stichwort Hammelsprung, in: Hans Röhring / Kurt Sontheimer (Hrsg.), a.a.O., S. 190; Bernd Leyendecker, Ab Ostern stimmt der Bundestag mit Knopfdruck ab, in: Das Parlament, 20. Jg. (1970), H. 7, S. 11. 
gramm auslösten. Bei Abstimmungen mussten die Abgeordneten sich aufrecht auf ihre Plätze setzen, bei ihren Anlagen eine Identitätsnummer einstellen und konnten dann zwischen „Ja“, „Nein“ und „Enthaltung“ wählen. Außerdem konnten sie bei Debatten ihren Redebedarf über das Gerät anmelden; dieses speicherte bis zu 20 Meldungen hintereinander und verschaffte dem Präsidenten einen leichteren Überblick über die Reihenfolge der Sprecher ${ }^{58}$. Allerdings kam es zu mehreren missglückten Einsätzen der Anlage ${ }^{59}$. Sie wurde in der 7. Wahlperiode stillgelegt und 1977 ausgebaut ${ }^{60}$.

\subsection{Ausblick}

In jüngster Zeit werden Forderungen erhoben, erneut einen Abstimmungscomputer im Bundestag zu verwenden, zuletzt nach Unregelmäßigkeiten bei der Abstimmung über den Entwurf des Haushaltsbegleitgesetzes 2006 am 19. Mai $2006^{61}$.

In anderen Parlamenten ist ein elektronisches Abstimmungsverfahren durchaus üblich, wobei die Details variieren. So sieht beispielsweise $\$ 70$ Abs. 4 der Geschäftsordnung des Berliner Abgeordnetenhauses eine elektronische Anlage vor. Art. 161 der Geschäftsordnung des Europäischen Parlaments regelt ebenfalls eine elektronische Abstimmung, wobei dort nur das ziffernmäßige Abstimmungsergebnis festgehalten wird. Nach Art. 56 des Geschäftsreglements des Schweizer Nationalrates erfolgt die Stimmabgabe sogar regelmäßig durch ein elektronisches Abstimmungssystem, wobei nach Art. 57 neben dem Resultat auch das Stimmverhalten der Nationalräte veröffentlicht wird.

$\mathrm{Ob}$ ein elektronisches Abstimmungssystem im Bundestag tatsächlich demnächst eingeführt wird, bleibt abzuwarten. Der Bundestag hat zuletzt während des Umzugs von Bonn nach Berlin das Thema diskutiert und sich gegen die Einführung eines solchen Systems gewandt. Insbesondere die nicht unerheblichen technischen Probleme (reibungsloses Funktionieren, Ausschluss von Fehlbenutzung oder Missbrauch) sowie die Kosten haben die Abgeordneten bisher dazu bewogen, sich gegen elektronische Abstimmungsverfahren zu entscheiden, zumal sich die etablierten Formen bewährt und keine nennenswerten Probleme verursacht haben ${ }^{62}$.

Auch der Bundesrat hat sich zum Einsatz einer elektronischen Abstimmungsanlage bisher nicht entschließen können, obwohl die technischen Möglichkeiten mittlerweile ausgefeilt sind ${ }^{63}$.

58 Bernd Leyendecker, a.a.O.

59 Zur letztmaligen Verwendung der Anlage siehe die Abstimmung vom 8. November 1973, Stenographische Protokolle Deutscher Bundestag, 7/64, S. 3725 f. Vgl. zum damaligen Innovationsbewusstsein Erbard Ulrich, Technikprognosen, in: MittAB, 13 Jg. (1980), S. 409.

60 Peter Schindler, 1999, a.a.O., S. 1727; Hans-Achim Roll, Geschäftsordnungspraxis im 10. Deutschen Bundestag, in: ZParl, 17. Jg. (1986), H. 3, S. 313 (S. 319).

61 Abg. Carl-Ludwig Thiele und Abg. Dagmar Enkelmann in BILD-online vom 31. Mai 2006. Zum Verlauf der Abstimmung am 19. Mai 2006 siehe Stenographische Protokolle Deutscher Bundestag, 16/36, S. 3116 (3139) und die Erklärung des Abg. Jochen-Konrad Fromme, ebenda, S. 3184.

62 Schreiben der Bundestagsverwaltung (Wissenschaftliche Dienste) vom Juli 2006 an Verfasser; siehe auch R. Peter Dach, in: Bonner Kommentar, a.a.O., Art. 40 Rn. 122 (Oktober 1996).

63 Vgl. Konrad Reuter, a.a.O., \$29 GO Rn. 3. 